\title{
Spiradiclis pengshuiensis (Ophiorrhizeae, Rubioideae), a new species from Chongqing, China
}

\author{
Bo Pan', Hu-Sheng Ma', Rui-Jiang Wang ${ }^{2}$ \\ I Guangxi Institute of Botany, Guangxi Zhuangzu Autonomous Region and Chinese Academy of Sciences, Gui- \\ lin 541006, China 2 Key Laboratory of Plant Resources Conservation and Sustainable Utilization, Guangdong \\ Provincial Key Laboratory of Applied Botany, South China Botanical Garden, Chinese Academy of Sciences, \\ Guangzhou 510650, China
}

Corresponding author: Rui-Jiang Wang (wangrj@scbg.ac.cn)

Academic editor: Pavel Stoev | Received 3 February 2016 | Accepted 14 May 2016 | Published 18 May 2016

Citation: Pan B, Ma H-S, Wang R-J (2016) Spiradiclis pengshuiensis (Ophiorrhizeae, Rubioideae), a new species from Chongqing, China. PhytoKeys 63: 41-45. doi: 10.3897/phytokeys.63.8016

\begin{abstract}
Spiradiclis pengshuiensis Bo Pan \& R. J. Wang (Rubiaceae) is described as a new species from Chongqing in SW China. It is morphologically compared with S. pauciflora L. Wu \& Q. R. Liu because of their similarities in habit, pubescent surface, small leaf laminas and subglobose capsules. Its conservation status is evaluated as "VU" according to the IUCN categories and criteria.
\end{abstract}

\section{Keywords}

China, New taxon, Rubiaceae, Spiradiclis

\section{Introduction}

The genus Spiradiclis Blume (Rubiaceae) mainly distributed in the tropical and subtropical limestone areas of Southern China and Northern Vietnam. Geographically, the localities of Spiradiclis species can extend northward to Mt. Emei $\left(29^{\circ} 33^{\prime} \mathrm{N}\right)$ in Sichuan province of China and southward to Central Java (ca. $7^{\circ} 10^{\prime} \mathrm{S}$ ) of Indonesia. The genus comprise about 50 species and ca. 91\% species can be found in China, the center of species diversity (Chen and Taylor 2011, Deng et al. 2014, Wang et al. 2015, Wen et al. 2015, Wu et al. 2015, Wang 2016). Spiradiclis species are usually similar to Ophiorrhiza L. because of their similar inflorescence and flower characters, 
but the former is characterized by globose or ovoid (vs. strongly laterally compressed in Ophiorrhiza) capsules.

During a botanical inventory in Pengshui County, the east of Chongqing Municipality, in 2013, the senior author found a striking Spiradiclis species growing on the dry cliffs. In order to get enough materials for morphological observation and comparison, we successively collected the vouchers during the flowering and fruiting seasons in recent years. This new species can be readily distinguished from other Spiradiclis species in habit and flowers and herein described and illustrated.

\section{Material and methods}

All materials were collected by ourselves and deposited at the herbarium of South China Botanical Garden, Chinese Academy of Sciences (IBSC). The morphological data were collected by a Digimatic Caliper (Mitutoyo, Japan).

\section{Taxonomy}

\section{Spiradiclis pengshuiensis B. Pan \& R. J. Wang, sp. nov.} urn:Isid:ipni.org:names:77154905-1

Figure 1

Diagnosis. Spiradiclis pengshuiensis is similar to S. pauciflora L. Wu \& Q. R. Liu, from which it differs in having linear (vs. triangular) stipules, 4-6 (vs. 3-4) secondary veins each side of the leaf laminas, salverform (vs. funnelform) corolla with 9-15 mm (vs. 7-9 $\mathrm{mm}$ ) long tubes (Table 1).

Type. CHINA. Chongqing Municipality: Pengshui County, Hanxia Town, Baixiang Village, $29^{\circ} 8^{\prime} \mathrm{N}, 108^{\circ} 6^{\prime} \mathrm{E}, 360 \mathrm{~m}$ alt., 29 Nov 2014, Rui-Jiang Wang 2931, longstyled flower (holotype IBSC; isotypes IBSC).

Description. Perennial herbs, 4-9 cm tall, densely pubescent on plant surfaces; stems erect or prostrate at base and then ascending, terete, rooting at nodes; inter nodes 2-15 mm long. Stipules linear, 0.9-1.2 mm long. Petiole (3.5-)7-12(-23.5) $\mathrm{mm}$ long. Leaf blades opposite, ovate, (4-)7-15(-25) $\times(2.5-) 5-10(-15) \mathrm{mm}$; base cuneate or late cuneate, asymmetry, decurrent; apex obtuse to rounded, papery, green adaxially and pale green abaxially; secondary veins $4-6$ on each side; margin revolute slightly. Inflorescence terminal, cymose, 1-7(-12)-flowered but only 1-3 blooming simultaneously; peduncles $1-2.1 \mathrm{~cm}$ long; bracts and bracteoles linear, 1-2 mm long. Flowers distylous; pedicels $0-2 \mathrm{~mm}$ long; hypanthium obconical, $1-2 \mathrm{~mm}$ long; calyx lobes 5 , lanceolate, $0.8-1.5 \times$ ca. $0.4 \mathrm{~mm}$. Corolla salverform, white adaxially, pinkish to white abaxially, pubescent both sides; tube 9-15 × 1.5-2 mm; lobes (4-)5, oval, 5-7.5 $\times 2.5-3.5 \mathrm{~mm}$. Stamens 5; anthers linear-oblong, 1.5-2 mm long, dorsi-fixed. Stigma bilobed, lobes ovoid; ovary 2-celled, ovules many, axile. Long-styled flowers: 
Table I. Morphological comparison of Spiradiclis pengshuiensis and S. pauciflora.

\begin{tabular}{|c|c|c|}
\hline Characters & S. pengshuiensis & S. pauciflora \\
\hline Habit & Erect or prostrate at base & Creeping or with upper parts ascending \\
\hline Indument & Pubescent whole plant surfaces & Pubescent whole plant surfaces \\
\hline Stem & Terete & Terete \\
\hline Stipules & Linear, $0.9-1.2 \mathrm{~mm}$ long & Triangular, less than $1 \mathrm{~mm}$ \\
\hline Petioles length $(\mathrm{mm})$ & $3.5-23.5$ & $3-10$ \\
\hline Leaf blades & $\begin{array}{l}\text { Ovate; base cuneate or late cuneate, } \\
\text { asymmetry, decurrent; apex obtuse to } \\
\text { rounded }\end{array}$ & $\begin{array}{l}\text { Ovate to elliptic-ovate; base obtuse to } \\
\text { broadly cuneate; apex obtuse to acute }\end{array}$ \\
\hline Leaf texture & Papery & Papery \\
\hline Leaf size $(\mathrm{mm})$ & $(4-) 7-15(-25) \times(2.5-) 5-10(-15)$ & $5-20 \times 5-15$ \\
\hline Leaf color & Green adaxially and pale green abaxially & $\begin{array}{l}\text { Olive-green adaxially, pale or sometime } \\
\text { purplish red abaxially }\end{array}$ \\
\hline $\begin{array}{l}\text { Secondary veins on } \\
\text { each side }\end{array}$ & $4-6$ & $3-4$ \\
\hline Inflorescence & Terminal, 1-7(-12)-flowered & Terminal, 3-7(-9)-flowered \\
\hline Peduncle length $(\mathrm{cm})$ & $1-2.1$ & $1-3$ \\
\hline Pedicel length $(\mathrm{mm})$ & $0-2$ & $0.3-3$ \\
\hline Calyx lobes & Lanceolate, $0.8-1.5 \mathrm{~mm}$ long & Ovate-triangular, $1.2-1.6 \mathrm{~mm}$ long \\
\hline Corolla & $\begin{array}{l}\text { Salverform; corolla tube } 9-15 \mathrm{~cm} \text { long; } \\
\text { lobes oval, } 5-7.5 \mathrm{~mm} \text { long }\end{array}$ & $\begin{array}{l}\text { Funnelform; corolla tube } 7-9 \mathrm{~mm} \text { long; } \\
\text { lobes ovate-triangular, } 2.5-3 \mathrm{~mm} \text { long }\end{array}$ \\
\hline Pin flowers & $\begin{array}{l}\text { Corolla pubescent adaxially, without ring } \\
\text { hairs; styles 9-12 } \mathrm{mm} \text { long }\end{array}$ & $\begin{array}{l}\text { Ring hairs present in adaxial side of corolla; } \\
\text { styles } 7.5-8.5 \mathrm{~mm} \text { long }\end{array}$ \\
\hline $\begin{array}{l}\text { Style length in thrum } \\
\text { flowers }(\mathrm{mm})\end{array}$ & ca. 5 & $2.7-3$ \\
\hline Capsules & Subglobose, $3-5 \mathrm{~mm}$ in diam. & Subglobose, ca. $2 \mathrm{~mm}$ in diam. \\
\hline Phenology & $\begin{array}{l}\text { Flowering in November to next January, } \\
\text { fruiting in December to February }\end{array}$ & $\begin{array}{l}\text { Flowering in March to June, fruiting in May } \\
\text { to August }\end{array}$ \\
\hline
\end{tabular}

stamens included; filaments adnate to the lower corolla tube, ca. $1.5 \mathrm{~mm}$ long; styles 9-12 mm long; stigma ca. $0.5 \mathrm{~mm}$ long, red to brown, extended to corolla throat, not exserted. Short-styled flowers: stamens extended to corolla throat, not exserted; filaments adnate to upper corolla tube, ca. $0.5 \mathrm{~mm}$ long; styles ca. $5 \mathrm{~mm}$ long; stigmas ca. $1.5 \mathrm{~mm}$ long, included. Capsules subglobose, $3-5 \mathrm{~mm}$ in diam., with persistent calyx lobes, dehiscent septicidally and loculicidally; valves 4, ovate, 3-5 mm long. Seeds 15-20 per capsule, ca. $0.5 \mathrm{~mm}$ long, brown, irregular pyramid to cuboid, foveolate on surface.

Phenology. Flowers from November to next January; fruits from December to next February.

Distribution and habitat. Spiradiclis pengshuiensis is known only from the type locality. Its habitat is on the cliffs nearby the A-Yi-He river but with very poor conditions.

Conservation status. Only three small populations and less than 500 individuals of Spiradiclis pengshuiensis within $5 \mathrm{~km}^{2}$ were found in the A-Yi-He Scenic Area. The tourist activity is the primary factor in the decline of populations. In addition, the 

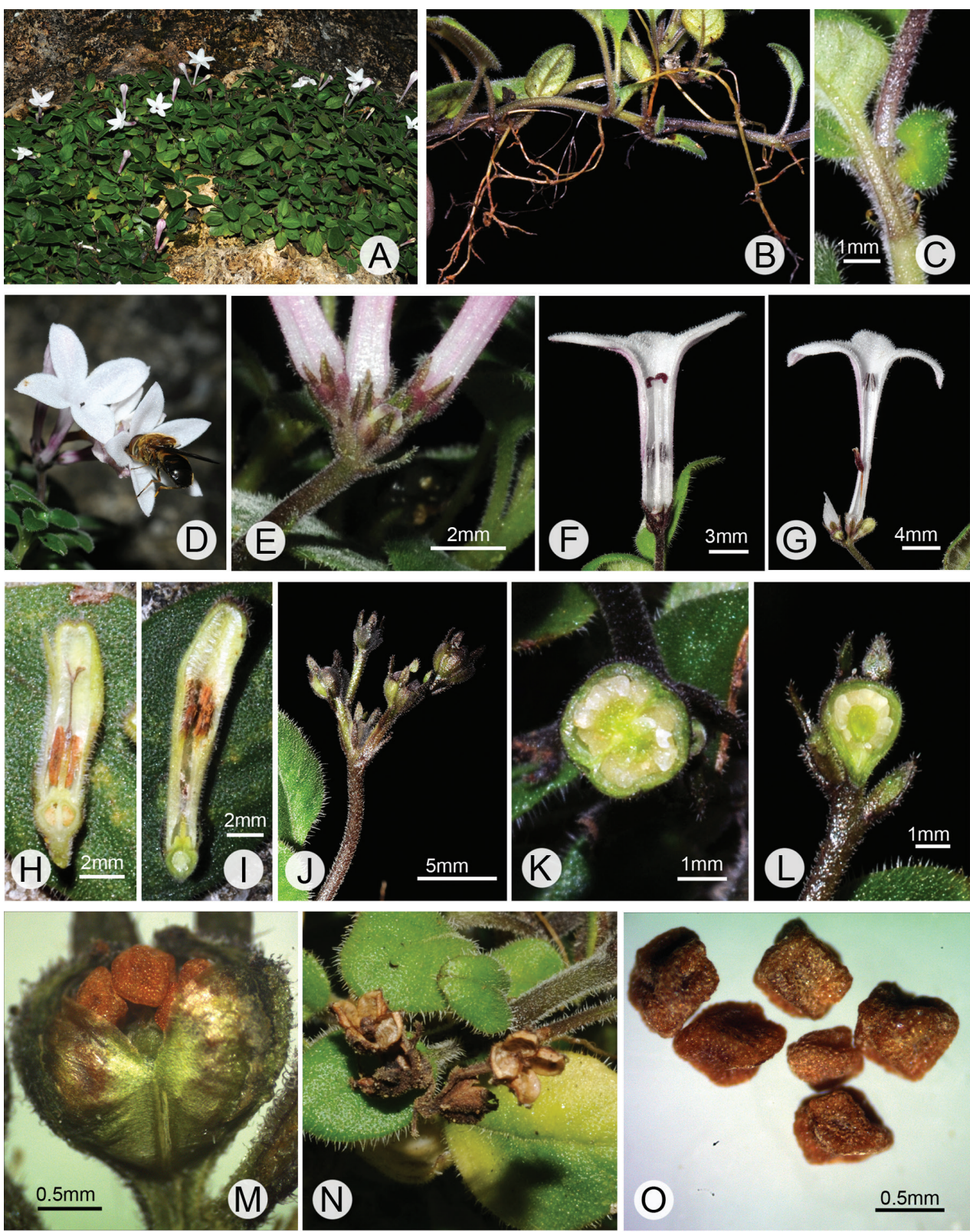

Figure I. Spiradiclis pengshuiensis sp. nov. A habitat $\mathbf{B}$ habit $\mathbf{C}$ linear stipules $\mathbf{D}$ visiting insects $\mathbf{E}$ bracts and hypanthium $\mathbf{F}-\mathbf{I}$ longitudinal section of long- $(\mathbf{F}, \mathbf{H})$ and short-styled flowers $(\mathbf{G}, \mathbf{I})$, respectively, showing the induments, relative positions and morphology of the stigmas and anthers and the developing capsules in distylous flowers $\mathbf{J}$ infructescence $\mathbf{K}-\mathbf{L}$ transverse and longitudinal section of young capsules, respectively $\mathbf{M}$ mature capsule $\mathbf{N}$ dehiscent capsules $\mathbf{O}$ seeds. Photos by Ruijiang Wang. 
plants always grow on the cliffs with little soil and insufficient water, which limited the development and dispersal of the species. Therefore we assign a preliminary IUCN threat status of Vulnerable [VU, B2ab(ii, iii, iv)] to S. pengshuiensis (IUCN 2001).

Specimens examined. CHINA. Chongqing Municipality: Pengshui County, Hanxia Town, Baixiang Village, $29^{\circ} 8^{\prime} \mathrm{N}, 108^{\circ} 6^{\prime} \mathrm{E}, 350 \mathrm{~m}$ alt., 29 Nov 2014, shortstyled flowers, Rui-Jiang Wang 2932, 2937 (IBSC); same locality, 12 Jan 2016, fruiting, Rui-Jiang Wang 3095, 3099 (IBSC).

\section{Acknowledgements}

This work was supported by National Natural Science Foundation of China (31070177) and Natural Science Foundation of Guangxi (2013GXNSFBA019078).

\section{References}

Chen T, Taylor CM (2011) Spiradiclis Blume. In: Wu ZY, Raven PH (Eds) Flora of China, vol. 19. Science Press, Beijing \& Missouri Botanical Garden Press, St. Louis, 330-339.

Deng SJ, Wen HZ, Huang XX, Wang RJ (2014) Spiradiclis coriaceifolia and S. tonglingensis spp. nov. (Rubiaceae, Ophiorrhizeae) from Guangxi, China. Nordic Journal of Botany 32: 594-601. doi: 10.1111/njb.00461

IUCN (2001) IUCN Red List Categories and Criteria (Version 3.1). IUCN Species Survival Commission, Gland, Switzerland and Cambridge. http://www.iucnredlist.org/info/categories_criteria2001

Wang RJ, Wen HZ, Deng SJ, Zhou LX (2015) Spiradiclis danxiashanensis (Rubiaceae), a new species from South China. Phytotaxa 206(1): 30-36. doi: 10.11646/phytotaxa.206.1.5

Wang RJ (2016) Spiradiclis yangchunensis (Rubiaceae), a new species from Guangdong, China. Plant Science Journal 34(1): 13-17. doi: 10.11913/PSJ.2095-0837.2016.10013

Wen HZ, Wang RJ, Deng SJ (2015) Spiradiclis longanensis, a new species of Rubiaceae from China. PhytoKeys 55: 113-117. doi: 10.3897/phytokeys.55.4975

Wu L, Wang JL, Liu QR (2015) Spiradiclis pauciflora (Rubiaceae), a new species from limestone areas in Guangxi, China. Annales Botanici Fennici 52(3-4): 257-261. doi: $10.5735 / 085.052 .0318$ 